\title{
Assessment of renal function using magnetic resonance quantitative histogram analysis based on spatial labeling with multiple inversion pulses
}

\author{
Ping Liang ${ }^{1}$, Shichao $\mathrm{Li}^{1}$, Chuou Xu${ }^{1}$, Jiali Li ${ }^{1}$, Fangqin Tan ${ }^{1}$, Daoyu $\mathrm{Hu}^{1}$, Ihab Kamel ${ }^{2}$, Zhen $\mathrm{Li}^{1}$ \\ ${ }^{1}$ Department of Radiology, Tongji Hospital, Tongji Medical College, Huazhong University of Science and Technology, Wuhan, China; ${ }^{2}$ Department \\ of Radiology and Radiological Science, The Johns Hopkins Hospital, Baltimore, MD, USA \\ Contributions: (I) Conception and design: Z Li, C Xu, I Kamel; (II) Administrative support: D Hu; (III) Provision of study materials or patients: F \\ Tan; (IV) Collection and assembly of data: S Li, J Li; (V) Data analysis and interpretation: P Liang; (VI) Manuscript writing: All authors; (VII) Final \\ approval of manuscript: All authors. \\ Correspondence to: Zhen Li. Department of Radiology, Tongji Hospital, Tongji Medical College, Huazhong University of Science and Technology, \\ 1095 Jiefang Avenue, Wuhan 430030, China. Email: zhenli@hust.edu.cn.
}

Background: The incidence of chronic kidney disease (CKD) is high, and is easy to develop into endstage renal disease (ESRD), which requires kidney dialysis or kidney transplantation. Therefore, we want to explore the clinical value of magnetic resonance quantitative histogram analysis based on spatial labeling with multiple inversion pulses (SLEEK) in assessing renal function in the early stage.

Methods: One hundred and twenty-nine patients underwent abdominal MRI examination, including a coronal SLEEK sequence. The patients were divided into the control group [CG, 47 cases, estimated glomerular filtration rate (eGFR) >90], the mild renal function impairment (mRI) group (48 cases, eGFR $=60-90)$, and the moderate to severe renal function impairment (m-sRI) group $(34$ cases, eGFR $<60)$. Two experienced radiologists delineated cortex and medulla regions of interest (ROIs) on SLEEK images to obtain cortex and medulla quantitative histogram parameters [Mean, Median, Percentiles $\left(5^{\text {th }}, 10^{\text {th }}, 25^{\text {th }}, 75^{\text {th }}\right.$, and $90^{\text {th }}$ ), Skewness, Kurtosis, and Entropy] using FireVoxel. These histogram parameters were compared by proper statistical methods such as one-way analysis of variance, the $\chi^{2}$ test, and receiver operating characteristic (ROC) curve analysis.

Results: Four histogram parameters (Inhomogeneity cortex, Skewness $_{\text {cortex }}$, Kurtosis medulla $_{\text {, and Entropy }}$ medulla) differed significantly between the CG and the mRI group. One medulla (Entropy medulla $_{\text {) }}$ and nine cortex $\left(\right.$ Mean $_{\text {cortex }}$, Median $_{\text {cortex }}$, Kurtosis $_{\text {cortex }}$, Entropy $_{\text {cortex }}$, and $5^{\text {th }}, 10^{\text {th }}, 25^{\text {th }}, 75^{\text {th }}$, and $90^{\text {th }}$ Percentile $\left._{\text {cortex }}\right)$ histogram parameters were significantly different between the m-RI and m-sRI groups. The most relevant parameter to eGFR was Inhomogenity cortex $_{(}(\mathrm{r}=-0.450, \mathrm{P}<0.001)$. Inhomogeneity ${ }_{\text {cortex }}$ had the largest area under the curve (AUC) for differentiating the mRI group from the CG (AUC $=0.718 ; 95 \%$ CI: 0.616-0.806), while $25^{\text {th }}$ Percentile $_{\text {cortex }}$ generated the largest AUC (AUC $=0.786$; 95\% CI: 0.681-0.869) for differentiating the mRI and m-sRI groups.

Conclusions: Quantitative histogram parameters based on a SLEEK sequence can be used to supplement renal dysfunction assessment. Cortex histogram parameters are more valuable for evaluating renal function than medulla histogram parameters.

Keywords: Histogram analysis; magnetic resonance imaging; renal function

Submitted May 04, 2021. Accepted for publication Aug 15, 2021.

doi: 10.21037/atm-21-2299

View this article at: https://dx.doi.org/10.21037/atm-21-2299 


\section{Introduction}

Chronic kidney disease (CKD) has been recognized as a major public health problem worldwide, with an estimated global prevalence of $13.4 \%$ (11.7-15.1\%) (1). Before the advanced stage of CKD, patients usually have no obvious symptoms. In clinical practice, the most common method for assessing kidney function is an estimation of the glomerular filtration rate, which reflects overall renal function and is susceptible to influence from various factors (2). When renal function is only slightly impaired, changes in the estimated glomerular filtration rate (eGFR) are not significant (3). As the disease progresses, patients can eventually develop end-stage renal disease (ESRD), the treatments for which include dialysis and transplantation (4). Therefore, it is critical to evaluate renal function in the early stages of CKD.

With the decline of renal function, the $\mathrm{T} 1$ relaxation time of the renal cortex and medulla are prolonged, which leads to a loss of corticomedullary differentiation, mainly in the cortex $(5,6)$. Due to microstructure and vascular distribution differences, the pathological changes and disease progression in the cortex and medulla differ. For example, most arterial blood flows directly into the renal cortex, with little reaching the medulla. Therefore, accurate differentiation of the renal cortex from the medulla is essential, especially in patients with renal insufficiency.

MRI technology has developed rapidly in the past few decades, and many imaging techniques, including intravoxel incoherent motion (IVIM) imaging, blood oxygenation level-dependent (BOLD) imaging, T1 mapping, and T2 mapping (7-11), have achieved good results in the assessment of renal function. However, previous studies did not analyze the renal cortex and medulla as two distinct regions of interest (ROIs).

Non-contrast enhanced MRI using spatial labeling with multiple inversion pulses (SLEEK) is a novel imaging technology that has made substantial progress in detecting renal arterial stenosis without a contrast agent (12). This imaging technique uses a flipping strip parallel to the long axis of the human body, which flips all soft tissue and blood signals in the coverage area, and another inverted strip placed horizontally above the kidney, which turns arterial blood into unsaturated blood again. A previous study found that SLEEK could display the renal artery by preparing multiple space selective inversion recovery pulses to label the blood flow in a more flexible way (13). Further, the optimal blood suppression inversion time in SLEEK was found to improve the corticomedullary contrast ratio of patients with renal insufficiency and healthy volunteers, enabling accurate measurement of renal cortical thickness to further assess renal function without contrast agents (14-16).

Quantitative histogram analysis is an emerging method for extracting high-throughput quantitative imaging features from CT/MRI images and subsequent analysis and interpretation of these data (17). Histogram analysis has made great progress in assessing lesion and organ heterogeneity, such as differentiating benign from malignant tumors, evaluating tumor grade, and predicting prognosis and clinical outcomes in some tumor types (18-20). Over recent decades, quantitative histogram analysis has been widely used to assess tumor heterogeneity $(21,22)$, and it also has numerous non-oncologic applications, including quantification of hepatic and renal fibrosis (23-25). Previous research has demonstrated that quantitative histogram analysis based on diffusion-weighted imaging (DWI), blood oxygen level-dependent MRI (BOLD), and susceptibility-weighted imaging (SWI) can be applied to evaluate renal dysfunction (26). However, in this study, ROIs were delineated around the outline of the whole renal parenchyma. To our knowledge, the renal cortex and medulla play different roles in renal function. Therefore, if we can develop a method to accurately distinguish the renal cortex and medulla and perform quantitative histogram analysis on the renal cortex and medulla individually, it may be possible to improve the accuracy and reliability of assessing renal function.

To the best of our knowledge, no prior studies have combined quantitative histogram analysis and SLEEK to assess renal function. This study explored the clinical value of magnetic resonance quantitative histogram analysis based on a SLEEK sequence in renal function assessment.

The following article is presented in accordance with the STARD reporting checklist (available at https://dx.doi. org/10.21037/atm-21-2299).

\section{Methods}

\section{Patients}

This retrospective study was conducted in accordance with the Declaration of Helsinki (as revised in 2013). The study was approved by the Institutional Review Board of Tongji Medical College, Huazhong University of Science and Technology (ethics approval ID: 2019S1035). The 


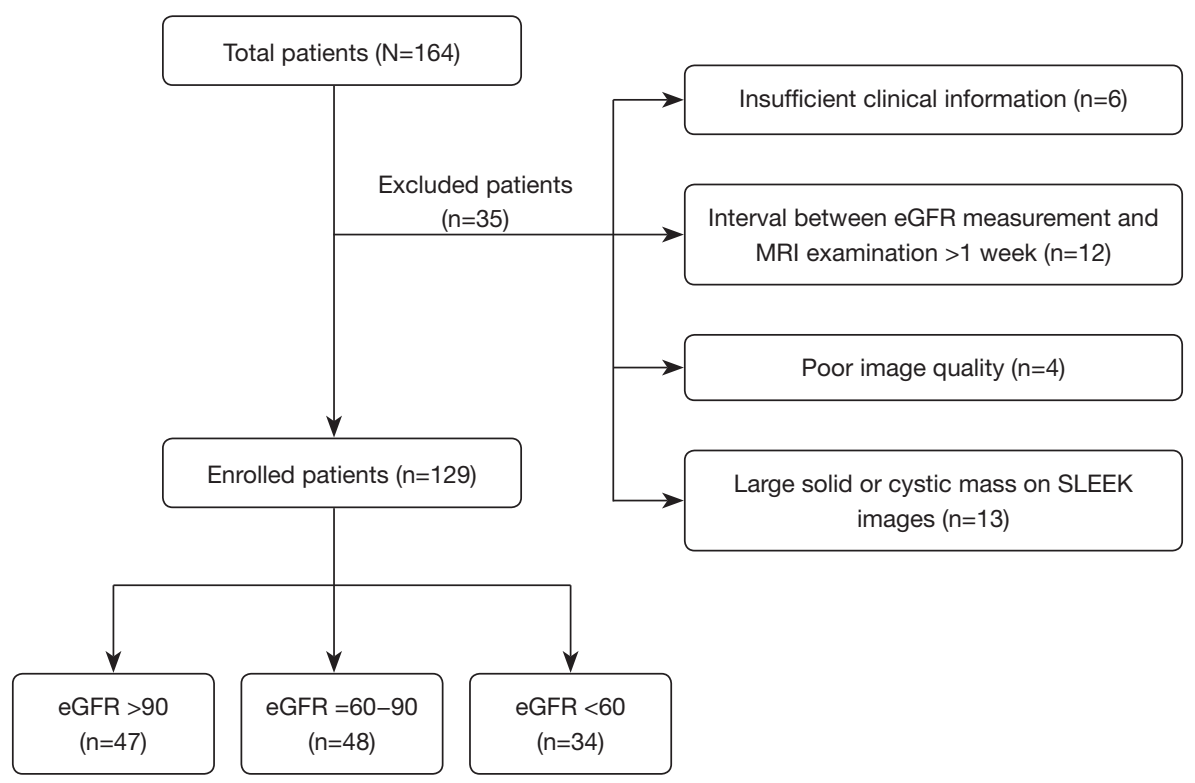

Figure 1 Flowchart of the study population selection.

requirement for individual consent was waived due to the study's retrospective nature.

We retrospectively analyzed our hospital radiology information system and identified 186 patients with or without renal insufficiency treated between January 2014 and October 2019. The most common clinical diseases among patients who underwent abdominal SLEEK examination were hypertension with suspected renal artery stenosis, atherosclerotic renal artery stenosis, chronic renal insufficiency, and various glomerular diseases. Twenty-two patients were excluded due to the absence of SLEEK images in the picture archiving and communication system (PACS). Two radiologists analyzed all images with 7 and 17 years of experience in abdominal imaging, and any discrepancies in image analysis between the two radiologists were resolved by consensus. A further 35 patients were excluded for the following reasons: (I) large solid/cystic lesion in the kidney; (II) insufficient clinical information; (III) poor-quality SLEEK images; (IV) an interval time of more than 1 week between eGFR measurement and MRI examination. The details of excluded patients are shown in Figure 1.

Finally, 129 patients (83 males and 46 females; age range, $17-85$ years; mean age, $51.79 \pm 13.8$ years) were included in the study. The eGFR value was calculated by using the modification of diet in renal disease (MDRD) formula (27) (Scr: serum creatinine):

$$
\begin{aligned}
e G F R\left(\mathrm{~mL} / \mathrm{min} / 1.73 \mathrm{~m}^{2}\right)= & 186 \times(\mathrm{Scr})^{-1.154} \times(\text { Age })^{-0.203} \\
& \times(0.742 \text { if female }) \\
& \times(1.210 \text { if African American })
\end{aligned}
$$

Study participants were classified in line with the CKD system in routine clinical use and were divided into control group (CG, 47 cases, eGFR $>90$ ), the mild renal function impairment (mRI) group (48 cases, eGFR $=60-90$ ), and the moderate to severe renal function impairment (m-sRI) group (34 cases, eGFR <60).

\section{MRI acquisition}

All examinations were performed on a $1.5 \mathrm{~T}$ system (Brivo MR360, GE Healthcare) with an eight-channel phasedarray coil. All patients were instructed to fast for 4 hours before the MRI examination. Conventional axial T1weighted (T1WI) imaging, T2-weighted (T2WI) imaging, BH Cor 2D FIESTA (breath-hold coronal two-dimensional fast imaging employing steady-state acquisition), and SLEEK were performed.

SLEEK is a respiratory-triggered three-dimensional fat-saturated fast imaging method employing steadystate acquisition prepared with multiple spatial selective inversion recovery pulses. Two orthogonal SLEEK bands 

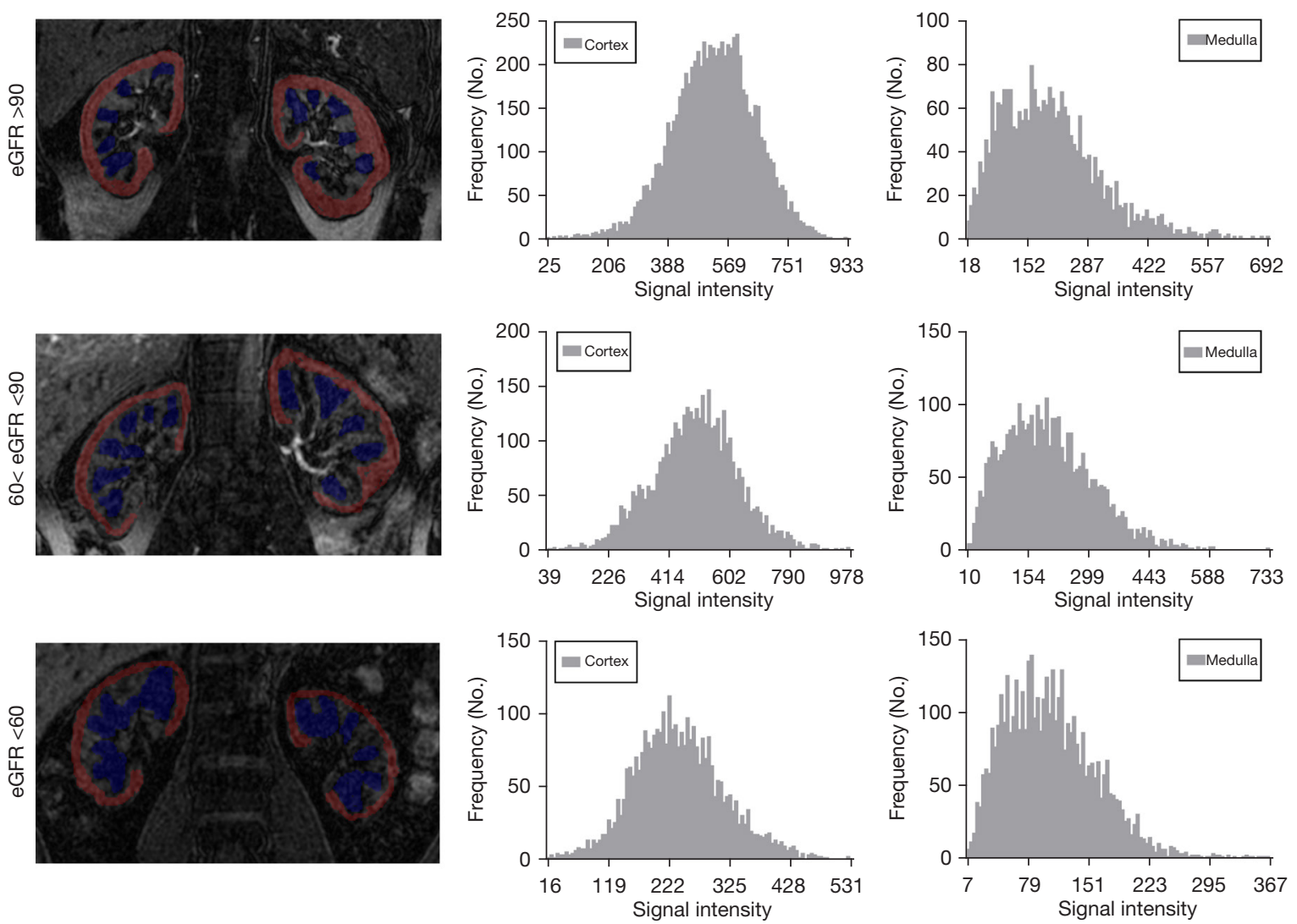

Figure 2 Representative ROIs on SLEEK images and corresponding gray-level histograms of the renal cortex and medulla of patients with eGFR >90, eGFR 60-90, and eGFR <60. ROIs, regions of interest; eGFR, estimated glomerular filtration rate.

were geographically localized to capture renal vessels based on the inflow effect. As described by Tang et al. (13), the SLEEK imaging technology uses two flipping strips to display the renal arteries, one is placed parallel to the long axis of the human body, and the other is placed above the kidney. To ensure the quality of the images, all patients underwent respiratory training before the examination, and based on our previous experience (12), the optimal blood suppression inversion time was $1,000 \mathrm{~ms}$. The SLEEK imaging parameters were as follows: repetition time/echo time $(\mathrm{TR} / \mathrm{TE})=5.01 / 2.50 \mathrm{~ms}$; number of acquisitions $=1$; parallel imaging factor $=2$; flip angle $=70$; receiver bandwidth $=125 \mathrm{~Hz} /$ pixel; slice thickness $=2 \mathrm{~mm}$; field of view $=600 \times 380 \mathrm{~mm}$; acquisition matrix $=256 \times 256$. Spatial labeling with multiple pulses (thickness of $130 \mathrm{~mm}$ ) was set on both kidneys. The SLEEK acquisition time ranged from 3 to 4 minutes, depending on the patient's respiration rate.

\section{Imaging analysis}

All original SLEEK images of the patients were transferred from the PACS to a PC. The two radiologists used a software package (Fire Voxel, New York University) to obtain the MRI histogram parameters of the renal cortex and medulla without clinical information.

The largest layer through the renal hilum was selected as the most representative layer for obtaining freehand ROIs of the renal cortex and medulla in the bilateral kidneys. The cortical ROI was delineated around the outline of the kidney, and 3-6 ROIs were placed on the medulla at the same level. All ROIs were placed to avoid perirenal fat, major blood vessels, and lesions, and they were saved on our computer for further histogram analysis.

Quantitative histogram parameters in the grayscale histogram included Mean, Median, Percentiles $\left(10^{\text {th }}, 25^{\text {th }}\right.$, 
Table 1 Basic clinical characteristics of the study participants

\begin{tabular}{lcccc}
\hline Characteristics & CG & mRl & m-sRl & P value \\
\hline Sex & 31 & 30 & 22 & 0.939 \\
Male & 16 & 18 & 12 & \\
Female & $51.15 \pm 10.10$ & $55.81 \pm 13.97$ & $51.44 \pm 11.57$ & 0.121 \\
Age (year) & $65.09 \pm 11.24$ & $86.09 \pm 15.05$ & $178.71 \pm 94.64$ & $<0.001$ \\
Serum creatinine $(\mu \mathrm{mol} / \mathrm{L})$ & $106.52 \pm 12.30$ & $76.69 \pm 7.85$ & $40.71 \pm 13.42$ & $<0.001$ \\
eGFR* & $7.39 \pm 0.60$ & $6.06 \pm 0.64$ & $4.46 \pm 0.75$ & $<0.001$ \\
Cortical thickness $(\mathrm{mm})$ & & &
\end{tabular}

*, the unit of eGFR is $\mathrm{mL} / \mathrm{min} / 1.73 \mathrm{~m}^{2}$. eGFR, estimated glomerular filtration rate; $\mathrm{CG}$, normal renal function; mRI, mild renal function impairment; $\mathrm{m}$-sRI, moderate to severe renal function impairment.

$75^{\text {th }}$, and $\left.90^{\text {th }}\right)$, Kurtosis, Skewness, and Entropy. The delineated ROIs and histogram analysis of the renal cortex and medulla are shown in Figure 2. Using the ruler tool in the Report Information System (RIS), the two radiologists measured the cortical thickness at the upper pole, hilar level, and lower pole of the bilateral kidneys. The average value of the six measurements was taken as the final result for statistical analysis. The post-processing of images took 3-4 minutes for each patient.

\section{Clinical data}

Clinical data, including sex, age, serum creatinine levels, and eGFR value, were recorded in our hospital's RIS system. Routine blood tests including serum creatinine and eGFR were performed within 1 week of the MRI examination. The study participants were divided into three groups based on their eGFR values. Serum creatinine levels exceeding $106 \mu \mathrm{mol} / \mathrm{L}$ are considered abnormal.

\section{Statistical analysis}

Statistical analyses were performed with SPSS version 22 (Chicago, IL, USA) and MedCalc (MedCalc Software, Mariakerke, Belgium). The significance of continuous variables between three groups was determined by oneway analysis of variance (ANOVA) or Kruskal-Wallis test. The two relevant groups were further compared using the least significant difference (LSD) test if a significant difference was found. Comparisons of categorical variables were conducted using the $\chi^{2}$ test. The Pearson or Spearman correlation was used to evaluate correlations between histogram parameters and eGFR. For correlation coefficients, an absolute value of below 0.3 indicated the absence of linear correlation, and an absolute value of above 0.3 indicated linear correlation, with values of $0.3-0.5$, $0.5-0.8$, and above 0.8 representing low, moderate, and high correlation, respectively. The diagnostic performances of the histogram parameters for differentiating the mRI group from the CG or m-sRI group were assessed by calculating the area under the curve (AUC) of the receiver operating characteristic (ROC) curve. The interobserver agreement of histogram parameters between the two radiologists was determined by calculating the interclass correlation coefficient (ICC) (excellent agreement, 0.81-1.00; moderate agreement, 0.61-0.80; fair agreement, 0.21-0.40; poor agreement, $0.00-0.20) . \mathrm{P}<0.05$ was considered statistically significant.

\section{Results}

\section{Comparison of patient characteristics}

One hundred and twenty-nine patients were included in the statistical analysis. The clinical characteristics of the study participants are shown in Table 1. There were no significant differences in sex or age between the three groups (chisquare $=0.126, \mathrm{P}=0.939),(\mathrm{F}=2.147, \mathrm{P}=0.121)$. However, serum creatinine, eGFR, and renal cortical thickness showed significant differences between three groups $(\mathrm{F}=54.84, \mathrm{P}=0.000 ; \mathrm{F}=341.16, \mathrm{P}=0.000 ; \mathrm{F}=196.11, \mathrm{P}=0.000$, respectively). Further, the renal cortical thickness was significantly positively correlated with the eGFR ( $r=0.741$, $\mathrm{P}<0.001)$. The results of the renal cortical thickness analysis are shown in Figure 3. 

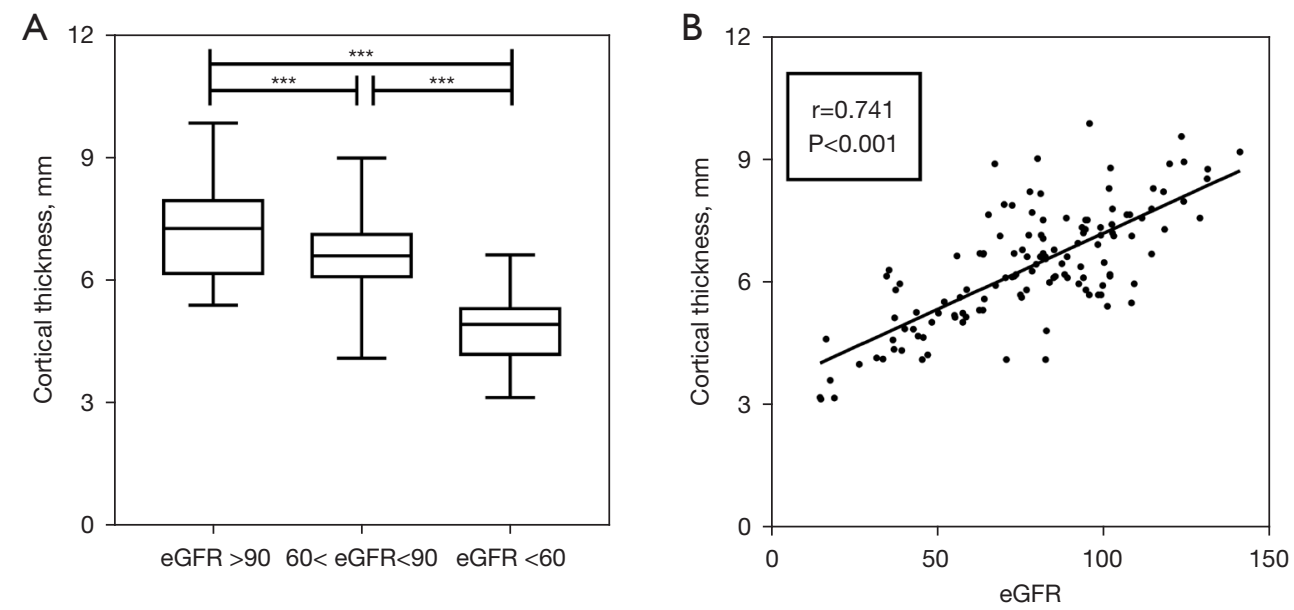

Figure 3 Analysis of the relationship between renal cortical thickness and the eGFR. Comparison of renal cortical thickness between the three groups of patients (A). The relationship between renal cortical thickness and the eGFR (B). ***, P<0.001, " $r$ " represents the correlation coefficient. The unit of eGFR is $\mathrm{mL} / \mathrm{min} / 1.73 \mathrm{~m}^{2}$. eGFR, estimated glomerular filtration rate.

Table 2 The interobserver agreement between two radiologists for different histogram parameters of SLEEK in the cortex and medulla

\begin{tabular}{lcccc}
\hline \multirow{2}{*}{ Parameters } & \multicolumn{2}{c}{ Intraclass correlation coefficient } & \multicolumn{2}{c}{$95 \%$ confidence interval } \\
\cline { 2 - 4 } \cline { 3 - 5 } Mean & Cortex & Medulla & Cortex & $0.990-0.995$ \\
Median & 0.998 & 0.993 & $0.998-0.999$ & $0.991-0.995$ \\
$10^{\text {th }}$ percentile & 0.998 & 0.994 & $0.998-0.999$ & $0.974-0.987$ \\
$25^{\text {th }}$ percentile & 0.996 & 0.982 & $0.994-0.997$ & $0.986-0.993$ \\
$75^{\text {th }}$ percentile & 0.997 & 0.990 & $0.996-0.998$ & $0.991-0.995$ \\
$90^{\text {th }}$ percentile & 0.999 & 0.994 & $0.998-0.999$ & $0.986-0.993$ \\
Inhomogeneity & 0.999 & 0.990 & $0.998-0.999$ & $0.967-0.983$ \\
Skewness & 0.975 & 0.977 & $0.964-0.982$ & $0.900-0.950$ \\
Kurtosis & 0.881 & 0.929 & $0.832-0.916$ & $0.713-0.915$ \\
Entropy & 0.738 & 0.814 & $0.630-0.815$ & $0.841-0.933$ \\
\hline
\end{tabular}

\section{Interobserver agreement}

The interobserver agreement was moderate to excellent for all histogram parameters, with ICCs ranging from 0.739 to 0.999 , reflecting good repeatability. Subsequently, a set of data measured by one of the radiologists was randomly selected for the statistical analysis. The ICC values for the histogram parameters of the cortex and medulla are shown in Table 2.

\section{Comparison of histogram parameters between the three groups}

Several histogram parameters (Inhomogeneity ${ }_{\text {cortex }}$, Skewness $_{\text {cortex }}$, Kurtosis medulla, and Entropy $_{\text {medulla }}$ ) showed a significant difference between the CG and $\mathrm{mRI}$ group (all $\mathrm{P}<0.05)$. The majority of cortex histogram parameters $\left(\right.$ Mean $_{\text {cortex }}$, Median $_{\text {cortex }}, 10^{\text {th }}$ Percentile $_{\text {cortex }}$, $25^{\text {th }}$ Percentile $_{\text {cortex }}, 75^{\text {th }}$ Percentile $_{\text {cortex }}, 90^{\text {th }}$ Percentile $_{\text {cortex }}$, 


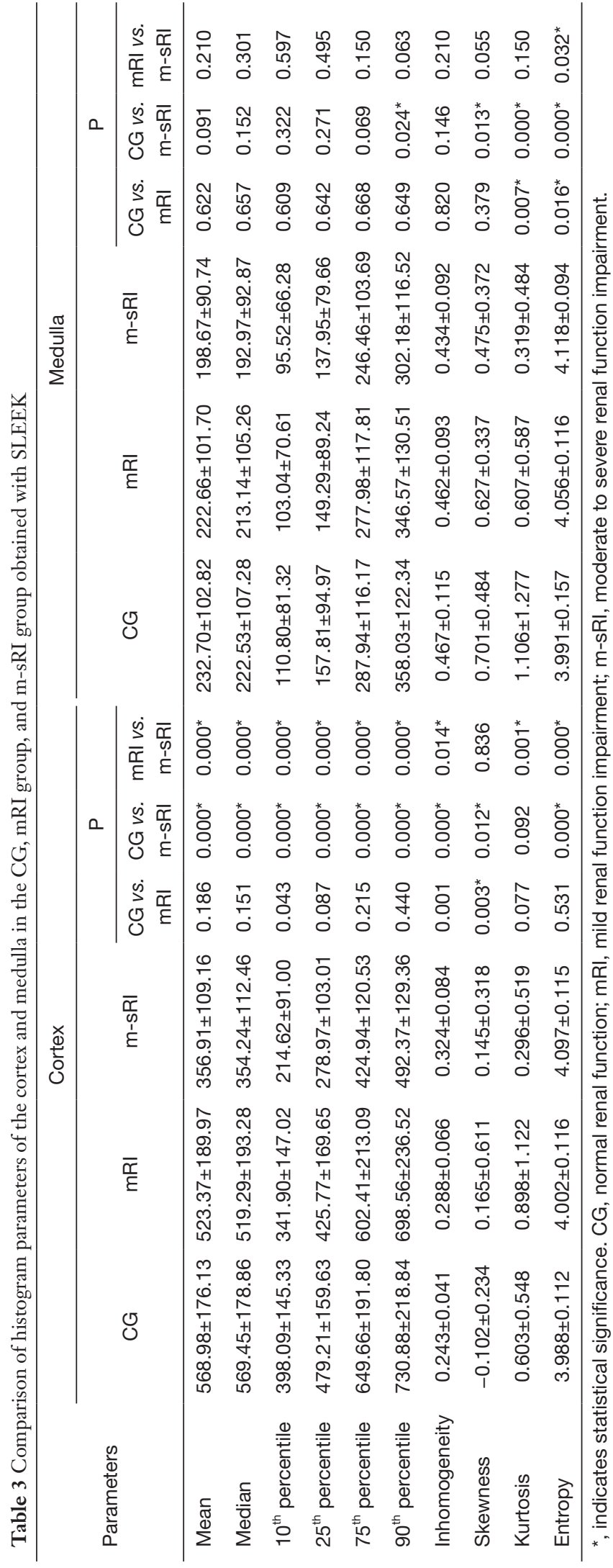

Inhomogeneity $_{\text {cortex }}$, Kurtosis cortex $_{\text {, and Entropy }}$ cortex $)$ demonstrated significant differences between the mRI and $\mathrm{m}$-sRI groups (all $\mathrm{P}<0.05$ ); however, only one medulla histogram parameter (Entropy medulla $_{\text {a }}$ ) showed a significant difference between the two groups $(\mathrm{P}<0.05)$. The results of comparisons between the three groups are shown in Table 3 and Figure 4.

\section{Correlation between histogram parameters and eGFR}

The correlations between eGFR and the renal cortex and medulla histogram parameters are presented in Figure 5. Of the renal cortex histogram parameters, Inhomogenity ${ }_{\text {cortex }}$ had the highest correlation coefficient with the eGFR $(\mathrm{r}=-0.450, \mathrm{P}<0.001)$. Only one medulla histogram parameter $\left(\right.$ Entropy $\left._{\text {medulla }}\right)$ was significantly negatively correlated with the eGFR $(r=-0.333, P<0.001)$. The correlation coefficients and $\mathrm{P}$ values between these histogram parameters and the eGFR are presented in Table 4.

\section{The capacity of histogram parameters to differentiate the $m R I$ group from the CG or m-sRI group}

The differences in medulla histogram parameters were not significant between the mRI group and the CG and m-sRI group, and only one medulla histogram parameter $($ Entropy medulla $)$ was slightly correlated with eGFR. Therefore, only cortex histogram parameters were included in the ROC curve analysis. Of the cortex histogram parameters, Inhomogeneity cortex $_{\text {had the largest AUC for }}$ distinguishing the mRI group from the CG $[\mathrm{AUC}=0.718$; $95 \%$ confidence interval $(\mathrm{CI}): 0.616-0.806]$, while the $25^{\text {th }}$ Percentile $_{\text {cortex }}$ had the largest AUC for differentiating the $\mathrm{mRI}$ and $\mathrm{m}$-sRI groups (AUC $=0.786$; 95\% CI: 0.6810.869).

Subsequently, we combined Inhomogeneity cortex $_{\text {and }}$ renal cortical thickness to differentiate the mRI group from the CG, and the combined AUC was 0.753 (95\% CI: 0.654-0.836). We also combined the $25^{\text {th }}$ Percentile $_{\text {cortex }}$ and renal cortical thickness to differentiate the mRI and m-sRI groups, and the combined AUC was 0.931 (95\% CI: $0.853-$ 0.975). The combined model improved the discrimination performance more than the use of the renal cortex histogram parameters alone. The ROC curves are presented in Figure 6, and the AUCs (95\% CI), cut-offs, sensitivity, and specificity of these cortex histogram parameters are shown in Table 5. 

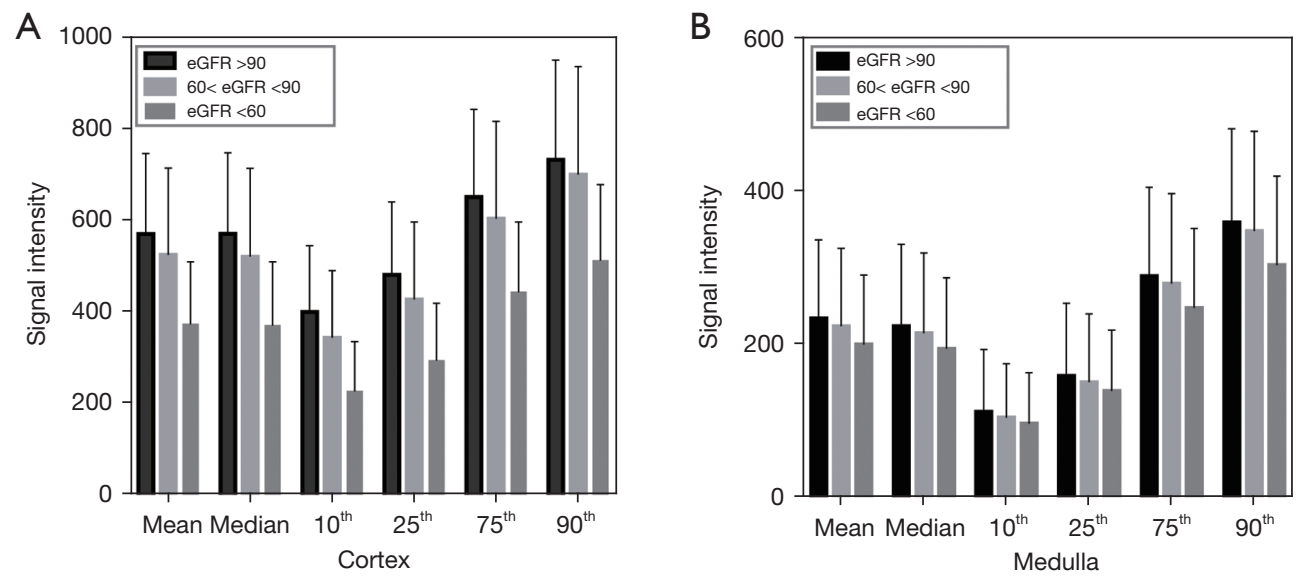

Figure 4 Bar charts comparing the SLEEK histogram parameters of renal cortex (A) and medulla (B) between the CG, mRI group, and m-sRI group. CG, control group; mRI, mild renal function impairment; m-sRI, moderate to severe renal function impairment.

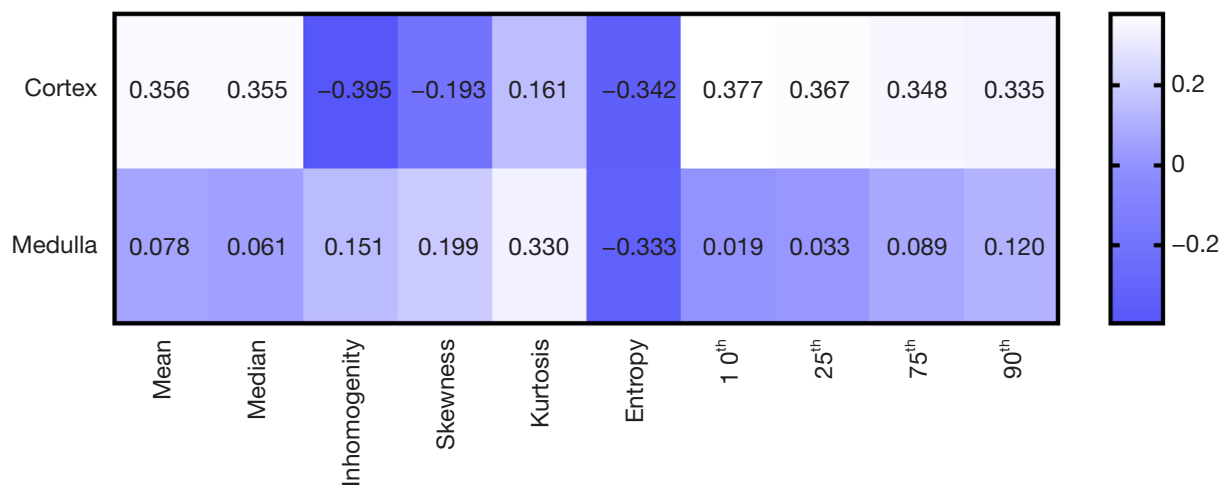

Figure 5 The relationship between the renal cortical and medullary histogram parameters and the eGFR. The first row shows the correlation between the renal cortical histogram parameters and eGFR, and the second row represents the correlation between the renal medulla histogram parameters and eGFR. eGFR, estimated glomerular filtration rate. eGFR, estimated glomerular filtration rate.

\section{Discussion}

This single-center study focused on the role of quantitative histogram analysis based on an MR SLEEK sequence in evaluating renal function. Our data demonstrated the potential of some quantitative histogram parameters obtained from the renal cortex and medulla to differentiate patients with $\mathrm{mRI}$ (eGFR $=60-90$ ) from those with normal renal function (CG, eGFR >90) or severe renal function impairment (m-sRI, eGFR <60). Compared to medulla histogram parameters, cortex histogram parameters might serve as more specific imaging biomarkers for the noninvasive evaluation of renal function.

For all volumetric quantitative histogram parameters, the interobserver agreement was excellent between the two radiologists, with the results showing good to excellent agreement overall. Excellent agreements demonstrated the good reliability and reproducibility of SLEEK histogram analysis.

A previous study indicated that the corticomedullary contrast ratio in SLEEK images showed no significant difference between the CG and mRI group (15), and the signal intensity changes of the renal parenchyma on SLEEK images were not noticeable when renal function declined slightly. However, in our study, Inhomogeneity cortex $_{\text {. }}$ and Skewness cortex $_{\text {showed significant differences between }}$ the CG and mRI groups. Inhomogeneity represents the heterogeneity of local tissues or complex pathological changes in lesions. As kidney function declines, the kidney 
tissue characteristics become more complex, including cellular swelling, fibrosis, inflammation, and perfusion reduction. Such changes increase the heterogeneity of kidney tissue. Skewness indicates an asymmetric signal strength distribution, and negative skew indicates that the tail on the left side of the histogram is longer than that on the right. Previous studies have shown that skewness can

Table 4 Correlation between eGFR and cortex and medulla histogram parameters obtained with SLEEK

\begin{tabular}{lcccccc}
\hline \multirow{2}{*}{ Parameters } & \multicolumn{2}{c}{ Cortex } & & \multicolumn{2}{c}{ Medulla } \\
\cline { 2 - 3 } \cline { 5 - 6 } \cline { 5 - 6 } Mean & $\mathrm{r}_{1}$ & $\mathrm{P}_{1}$ & & $\mathrm{r}_{2}$ & $\mathrm{P}_{2}$ \\
Median & 0.385 & $0.000^{*}$ & & 0.078 & 0.381 \\
$10^{\text {th }}$ percentile & 0.388 & $0.000^{*}$ & & 0.061 & 0.489 \\
$25^{\text {th }}$ percentile & 0.423 & $0.000^{*}$ & & 0.019 & 0.827 \\
$75^{\text {th }}$ percentile & 0.407 & $0.000^{*}$ & & 0.033 & 0.715 \\
$90^{\text {th }}$ percentile & 0.369 & $0.000^{*}$ & & 0.089 & 0.315 \\
Inhomogeneity & 0.347 & $0.000^{*}$ & & 0.120 & 0.176 \\
Entropy & -0.450 & $0.000^{*}$ & & 0.151 & 0.087 \\
\hline
\end{tabular}

${ }^{*}$, indicates statistical significance. $r_{1}$, represents the correlation coefficient between eGFR and histogram parameters of the cortex; $r_{2}$, represents the correlation coefficient between eGFR and histogram parameters of the medulla. eGFR, estimated glomerular filtration rate. aid in distinguishing tissues of different pathological types, including tumors (28). In the present study, the skewness of the mRI group was significantly higher than that of the CG, and the value of skewness was negative in the CG group. This finding was consistent with observations in previous research (29), and indicated that more voxels were distributed on the right side of the mean.

Quantitative histogram parameters obtained from the renal cortex, except for skewness, demonstrated a good diagnostic performance in differentiating $\mathrm{mRI}$ from m-sRI. However, apart from Kurtosis and Entropy, the renal medulla parameters were not statistically different between the patient groups. This may be attributable to the renal cortex being the most important part of kidney filtration and reabsorption and the fact that the main pathological changes in CKD mainly occur in the renal cortex. A previous study showed that the blood supply to the renal medulla is low and hypoxic under normal conditions (30). The renal medulla has been more sensitive to ischemia and hypoxia changes than the renal cortex (5). However, our study mainly focused on the changes in signal intensity when pathological changes occurred in the renal cortex and medulla. This may explain why most quantitative histogram parameters of the renal medulla in our study showed no significant difference between the three groups. These results demonstrated that SLEEK imaging may be more sensitive to renal cortex signal intensity changes and that

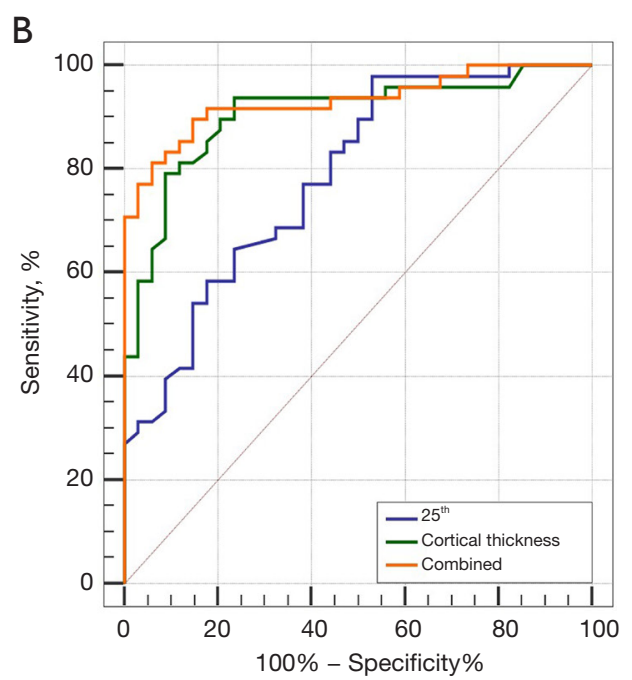

Figure 6 The ROC curve analysis of the Inhomogeneity cortex $_{2} 25^{\text {th }}$ Percentile $_{\text {cortex }}$, and renal cortical thickness to differentiate the mRI group from the CG (A) and sRI group (B). ROC, receiver operating characteristic; CG, control group; mRI, mild renal function impairment; sRI, severe renal function impairment. 
Table 5 Effectiveness of renal cortex histogram parameters obtained with SLEEK for discriminating the mRI group from the CG and m-sRI group

\begin{tabular}{|c|c|c|c|c|c|c|c|c|}
\hline Parameters & \multicolumn{4}{|c|}{$\mathrm{CG}$ and $\mathrm{mRI}$} & \multicolumn{4}{|c|}{$\mathrm{mRI}$ and $\mathrm{m}-\mathrm{sRI}$} \\
\hline Median & $0.604(0.498-0.702)$ & 470 & 52.08 & 72.34 & $0.780(0.675-0.864)$ & 440 & 85.29 & 64.58 \\
\hline $10^{\text {th }}$ percentile & $0.627(0.522-0.724)$ & 311 & 54.17 & 76.60 & $0.775(0.670-0.860)$ & 275 & 76.47 & 66.67 \\
\hline $75^{\text {th }}$ percentile & $0.592(0.487-0.692)$ & 489 & 33.33 & 87.23 & $0.771(0.664-0.856)$ & 505 & 82.35 & 66.67 \\
\hline $90^{\text {th }}$ percentile & $0.557(0.451-0.659)$ & 543 & 31.25 & 87.23 & $0.783(0.678-0.866)$ & 581 & 82.35 & 66.67 \\
\hline Inhomogeneity & $0.718(0.616-0.806)^{\star}$ & 0.263 & 60.42 & 72.34 & $0.622(0.508-0.727)$ & 0.277 & 50.00 & 76.47 \\
\hline Cortical thickness & $0.668(0.564-0.761)^{*}$ & 6.78 & 70.83 & 68.09 & $0.905(0.820-0.959)^{*}$ & 5.95 & 91.18 & 79.17 \\
\hline Combined & $0.753(0.654-0.836)^{*}$ & NS & 70.83 & 78.72 & $0.931(0.853-0.975)^{*}$ & NS & 81.25 & 94.12 \\
\hline
\end{tabular}

*, represents the three parameters with the largest AUC value. Combined means combined diagnosis of the two parameters with the highest AUCs. NS, not suitable. CG, normal renal function; mRI, mild renal function impairment; $\mathrm{m}$-sRI, moderate-severe renal function impairment; AUC, area under the receiver operating characteristic curve; $\mathrm{Cl}$, confidence interval.

the cortex is of greater clinical significance than the medulla in the study of renal insufficiency based on signal intensity changes. Interstitial fibrosis, ischemia, hypoxia, and capillary integrity around the renal tubules may be more relevant to the medulla than the cortex. BOLD MRI, which is more sensitive to changes in oxygen content (31), may be more suitable for further study of the pathological changes of the renal medulla in patients with renal insufficiency.

Quantitative histogram analysis is a quantitative and noninvasive method that can detect subtle changes in normal tissue or in lesions on CT/MRI images by quantitatively and objectively extracting relevant histogram features (17). The pixel-level distribution in the ROI can be evaluated and calculated by mathematical methods to assess oncologic and nononcologic pathological processes. On SLEEK images, lower percentiles $\left(10^{\text {th }}\right.$ and $\left.25^{\text {th }}\right)$ usually represent cystic or edema, whereas higher percentiles $\left(75^{\text {th }}\right.$ and $90^{\text {th }}$ ) represent higher blood perfusion. In our research, the values of lower percentiles $\left(10^{\text {th }}\right.$ and $\left.25^{\text {th }}\right)$ in the m-sRI group were significantly lower than those in the mRI group. This result was consistent with one reported in a previous study that showed the major pathological changes in the kidney when renal function declines to be fibrosis, edema, and inflammation (32). The values of higher percentiles $\left(75^{\text {th }}\right.$ and $\left.90^{\text {th }}\right)$ in the $\mathrm{m}$-sRI group were significantly lower than those in the mRI group, and a previous study indicated that renal cortex blood perfusion decreased with the decline of renal function (10). Kurtosis represents the peakedness of signal intensity distribution (23). A previous study demonstrated that histogram analysis could objectively assess the heterogeneity of the entire liver, especially kurtosis (23). In our study, Kurtosis cortex $_{\text {in the mRI }}$ group was significantly higher than that in the m-sRI group, which may be related to renal cortical fibrosis in patients with renal insufficiency. Entropy represents the irregularity of signal intensity distribution within a histogram on SLEEK images. In our study, Entropy cortex $_{\text {in the m-sRI }}$ group was significantly higher than that in the mRI group, and a significant negative correlation was observed between entropy and eGFR. This result was similar to the observation of a previous study that entropy increased with the increase in the fibrosis stage (33).

Most cortex histogram parameters (except for skewness and kurtosis) were related to eGFR, with Inhomogeneity cortex $_{\text {. }}$ having the strongest relationship with eGFR among all the histogram parameters. Further, the minimum renal cortical 
thickness exhibited significant differences between the three groups in our study, and the correlation coefficient between the minimum renal cortical thickness and eGFR was excellent. Combining the minimum renal cortical thickness and Inhomogeneity cortex $_{\text {or }} 25^{\text {th }}$ Percentile $_{\text {cortex }}$ can generate a higher AUC for differentiating patients with mRI from those with m-sRI or the CG. The minimum renal cortical thickness may be a valuable index and add precious value to the histogram parameters for evaluating the renal function, and this finding is similar to one of a previous study (15). Therefore, we can accurately measure the thickness of the renal cortex on SLEEK images and combine histogram parameters obtained from the renal cortex to assess renal function at an early stage.

There are several limitations to our study. First, it was a retrospective study, and there was a lack of internal and external validation due to the small number of patients. Second, we did not compare the SLEEK sequence with other functional sequences, such as DWI or BOLD. Third, we only applied TI $=1,000 \mathrm{~ms}$ in our study, and did not compare it with other TI values to obtain the best TI value showing better corticomedullary contrast ratio. Fourth, we only analyzed first-order parameters of MR histogram, and higher-order parameters will be included in our future research. Finally, our study did not include information on clinical variables, histology, or acute kidney injury status.

In conclusion, quantitative histogram parameters based on SLEEK, especially inhomogeneity cortex $_{\text {and }} 25^{\text {th }}$ Percentile $_{\text {cortex }}$, in combination with renal cortical thickness, may be suitable for assessing renal function in the early stages of renal insufficiency.

\section{Acknowledgments}

We would like to thank Pratik Tripathi for his help in polishing the paper.

Funding: This study received funding from the National Natural Science Foundation of China (Grant No. 81771801, 82071889).

\section{Footnote}

Reporting Checklist: The authors have completed the STARD reporting checklist. Available at https://dx.doi. org/10.21037/atm-21-2299

Data Sharing Statement: Available at https://dx.doi. org/10.21037/atm-21-2299
Peer Review File: Available at https://dx.doi.org/10.21037/ atm-21-2299

Conflicts of Interest: All authors have completed the ICMJE uniform disclosure form (available at https://dx.doi. org/10.21037/atm-21-2299). The authors have no conflicts of interest to declare.

Etbical Statement: The authors are accountable for all aspects of the work in ensuring that questions related to the accuracy or integrity of any part of the work are appropriately investigated and resolved. This retrospective study was conducted in accordance with the Declaration of Helsinki (as revised in 2013). The study was approved by the Institutional Review Board of Tongji Medical College, Huazhong University of Science and Technology (ethics approval ID: 2019S1035), and the requirement for individual consent for this retrospective analysis was waived.

Open Access Statement: This is an Open Access article distributed in accordance with the Creative Commons Attribution-NonCommercial-NoDerivs 4.0 International License (CC BY-NC-ND 4.0), which permits the noncommercial replication and distribution of the article with the strict proviso that no changes or edits are made and the original work is properly cited (including links to both the formal publication through the relevant DOI and the license). See: https://creativecommons.org/licenses/by-nc-nd/4.0/.

\section{References}

1. Lv JC, Zhang LX. Prevalence and Disease Burden of Chronic Kidney Disease. Adv Exp Med Biol 2019;1165:3-15.

2. Agarwal R, Delanaye P. Glomerular filtration rate: when to measure and in which patients? Nephrol Dial Transplant 2019;34:2001-7.

3. Levey AS, Eckfeldt JH. Estimating Glomerular Filtration Rate Using Serum Creatinine. Clin Chem 2017;63:1161-2.

4. Webster AC, Nagler EV, Morton RL, et al. Chronic Kidney Disease. Lancet 2017;389:1238-52.

5. Cox EF, Buchanan CE, Bradley CR, et al. Multiparametric Renal Magnetic Resonance Imaging: Validation, Interventions, and Alterations in Chronic Kidney Disease. Front Physiol 2017;8:696.

6. Lee VS, Kaur M, Bokacheva L, et al. What causes diminished corticomedullary differentiation in renal 
insufficiency? J Magn Reson Imaging 2007;25:790-5.

7. Mao W, Zhou J, Zeng M, et al. Chronic kidney disease: Pathological and functional evaluation with intravoxel incoherent motion diffusion-weighted imaging. J Magn Reson Imaging 2018;47:1251-9.

8. Milani B, Ansaloni A, Sousa-Guimaraes S, et al. Reduction of cortical oxygenation in chronic kidney disease: evidence obtained with a new analysis method of blood oxygenation level-dependent magnetic resonance imaging. Nephrol Dial Transplant 2017;32:2097-105.

9. Wolf M, de Boer A, Sharma K, et al. Magnetic resonance imaging T1- and T2-mapping to assess renal structure and function: a systematic review and statement paper. Nephrol Dial Transplant 2018;33:ii41-50.

10. Pruijm M, Milani B, Pivin E, et al. Reduced cortical oxygenation predicts a progressive decline of renal function in patients with chronic kidney disease. Kidney Int 2018;93:932-40.

11. Friedli I, Crowe LA, Berchtold L, et al. New Magnetic Resonance Imaging Index for Renal Fibrosis Assessment: A Comparison between Diffusion-Weighted Imaging and T1 Mapping with Histological Validation. Sci Rep 2016;6:30088.

12. Pei $Y$, Shen $\mathrm{H}, \mathrm{Li}$ J, et al. Evaluation of renal artery in hypertensive patients by unenhanced MR angiography using spatial labeling with multiple inversion pulses sequence and by CT angiography. AJR Am J Roentgenol 2012;199:1142-8.

13. Tang $H$, Wang $Z$, Wang $L$, et al. Depiction of transplant renal vascular anatomy and complications: unenhanced MR angiography by using spatial labeling with multiple inversion pulses. Radiology 2014;271:879-87.

14. Kanki A, Ito K, Tamada T, et al. Corticomedullary differentiation of the kidney: evaluation with noncontrastenhanced steady-state free precession (SSFP) MRI with time-spatial labeling inversion pulse (time-SLIP). J Magn Reson Imaging 2013;37:1178-81.

15. Noda Y, Ito K, Kanki A, et al. Measurement of renal cortical thickness using noncontrast-enhanced steadystate free precession MRI with spatially selective inversion recovery pulse: Association with renal function. J Magn Reson Imaging 2015;41:1615-21.

16. Liang $\mathrm{P}, \mathrm{Xu} \mathrm{C}$, Tripathi $\mathrm{P}$, et al. One-stop assessment of renal function and renal artery in hypertensive patients with suspected renal dysfunction: non-enhanced MRI using spatial labeling with multiple inversion pulses. Eur Radiol 2021;31:94-103.

17. Lubner MG, Smith AD, Sandrasegaran K, et al.
CT Texture Analysis: Definitions, Applications, Biologic Correlates, and Challenges. Radiographics 2017;37:1483-503.

18. Tian Z, Chen C, Fan Y, et al. Glioblastoma and Anaplastic Astrocytoma: Differentiation Using MRI Texture Analysis. Front Oncol 2019;9:876.

19. Li A, Xing W, Li H, et al. Subtype Differentiation of Small $(\leq 4 \mathrm{~cm})$ Solid Renal Mass Using Volumetric Histogram Analysis of DWI at 3-T MRI. AJR Am J Roentgenol 2018;211:614-23.

20. Choi IY, Yeom SK, Cha J, et al. Feasibility of using computed tomography texture analysis parameters as imaging biomarkers for predicting risk grade of gastrointestinal stromal tumors: comparison with visual inspection. Abdom Radiol (NY) 2019;44:2346-56.

21. Lu J, Hu D, Tang H, et al. Assessment of tumor heterogeneity: Differentiation of periampullary neoplasms based on CT whole-lesion histogram analysis. Eur J Radiol 2019;115:1-9.

22. Woo S, Cho JY, Kim SY, et al. Histogram analysis of apparent diffusion coefficient map of diffusion-weighted MRI in endometrial cancer: a preliminary correlation study with histological grade. Acta Radiol 2014;55:1270-7.

23. Zheng Y, Xu YS, Liu Z, et al. Whole-Liver Apparent Diffusion Coefficient Histogram Analysis for the Diagnosis and Staging of Liver Fibrosis. J Magn Reson Imaging 2020;51:1745-54.

24. Yu H, Buch K, Li B, et al. Utility of texture analysis for quantifying hepatic fibrosis on proton density MRI. J Magn Reson Imaging 2015;42:1259-65.

25. Zha T, Ren X, Xing Z, et al. Evaluating Renal Fibrosis with R2* Histogram Analysis of the Whole Cortex in a Unilateral Ureteral Obstruction Model. Acad Radiol 2019;26:e202-7.

26. Ding J, Xing Z, Jiang $Z$, et al. Evaluation of renal dysfunction using texture analysis based on DWI, BOLD, and susceptibility-weighted imaging. Eur Radiol 2019;29:2293-301.

27. Levey AS, Bosch JP, Lewis JB, et al. A more accurate method to estimate glomerular filtration rate from serum creatinine: a new prediction equation. Modification of Diet in Renal Disease Study Group. Ann Intern Med 1999;130:461-70.

28. Li J, Lu J, Liang P, et al. Differentiation of atypical pancreatic neuroendocrine tumors from pancreatic ductal adenocarcinomas: Using whole-tumor CT texture analysis as quantitative biomarkers. Cancer Med 2018;7:4924-31.

29. Li HM, Zhang R, Gu WY, et al. Whole solid tumour 
volume histogram analysis of the apparent diffusion coefficient for differentiating high-grade from lowgrade serous ovarian carcinoma: correlation with Ki-67 proliferation status. Clin Radiol 2019;74:918-25.

30. Ding J, Xing W, Wu D, et al. Evaluation of Renal Oxygenation Level Changes after Water Loading Using Susceptibility-Weighted Imaging and T2* Mapping. Korean J Radiol 2015;16:827-34.

31. Vakilzadeh N, Zanchi A, Milani B, et al. Acute hyperglycemia increases renal tissue oxygenation as measured by BOLD-MRI in healthy overweight

Cite this article as: Liang P, Li S, Xu C, Li J, Tan F, Hu D, Kamel I, Li Z. Assessment of renal function using magnetic resonance quantitative histogram analysis based on spatial labeling with multiple inversion pulses. Ann Transl Med 2021;9(21):1614. doi: 10.21037/atm-21-2299 volunteers. Diabetes Res Clin Pract 2019;150:138-43.

32. Wang W, Yu Y, Wen J, et al. Combination of Functional Magnetic Resonance Imaging and Histopathologic Analysis to Evaluate Interstitial Fibrosis in Kidney Allografts. Clin J Am Soc Nephrol 2019;14:1372-80.

33. Fujimoto K, Tonan T, Azuma S, et al. Evaluation of the mean and entropy of apparent diffusion coefficient values in chronic hepatitis $\mathrm{C}$ : correlation with pathologic fibrosis stage and inflammatory activity grade. Radiology 2011;258:739-48. 